• 研究报告・

\title{
中国沿海洛氏角毛藻复合群的 多样性组成及地理分布
}

\author{
陈作艺 ${ }^{1,2}$ 许晓静 ${ }^{1}$ 朱素英 ${ }^{1}$ 翟梦怡 ${ }^{1}$ 李 扬 ${ }^{*}$ \\ 1 (华南师范大学生命科学学院, 广州市亚热带生物多样性与环境生物监测重点实验室, \\ 广东省水产健康安全养殖重点实验室, 广州 510631) \\ 2 (河北省地矿局第八地质大队, 河北秦皇岛 066001)
}

\begin{abstract}
摘要: 洛氏角毛藻复合群(Chaetoceros lorenzianus complex)指具有与洛氏角毛藻相似形态学特征的物种集合, 它 们广泛分布于全球近岸水域。近年国际上关于该复合群的分类学研究取得新进展, 而我国相关研究仍较为滞后。 为了弄清我国沿海洛氏角毛藻复合群的物种多样性, 明确物种信息, 厘清种间界限, 为相关研究提供准确的物种 鉴定依据, 本研究陆续在中国沿海建立了该复合群的332个单克隆培养株系, 利用光学显微镜、扫描电镜和透射电 镜进行了较为详尽的形态学研究, 基于核糖体大亚基编码基因D1-D3区序列, 构建了分子系统学关系。结果表明 其形态聚类与分子系统学结论相一致, 显示我国洛氏角毛藻复合群具有较高的物种多样性, 共鉴定到 5 个物种, 分 别是并基角毛藻(C. decipiens)、优美角毛藻(C. elegans)、平狍角毛藻(C. laevisporus)、曼纳角毛藻(C. mannaii)和稀 树角毛藻(C. pauciramosus)。研究表明传统认知的光镜下特征, 如群体特征、角毛走势等易变化, 其分类学价值需 谨慎应用。角毛的超微结构, 如角毛孔纹的形状、大小、密度等是有效的种间区别特征, 休眠孢子亦是重要的物 种识别依据。并基角毛藻和平狍角毛藻在我国沿岸的分布范围最为广泛, 而稀树角毛藻的分布较为有限。
\end{abstract}

关键词：洛氏角毛藻复合群; 物种多样性; 形态学; 分子系统学; 休眠孢子; 中国沿海

\section{Species diversity and geographical distribution of the Chaetoceros lorenzianus complex along the coast of China}

\author{
Zuoyi Chen ${ }^{1,2}$, Xiaojing $\mathrm{Xu}^{1}$, Suying Zhu ${ }^{1}$, Mengyi Zhai ${ }^{1}$, Yang $\mathrm{Li}^{1 *}$ \\ 1 Guangzhou Key Laboratory of Subtropical Biodiversity and Biomonitoring, Guangdong Provincial Key Laboratory of \\ Healthy and Safe Aquaculture, School of Life Sciences, South China Normal University, Guangzhou 510631 \\ 2 The Eighth Geological Brigade, Hebei Geological Prospecting Bureau, Qinhuangdao, Hebei 066001
}

\begin{abstract}
The Chaetoceros lorenzianus complex is composed of several planktonic diatom species that share a similar morphology with C. lorenzianus Grunow. The complex has been recorded frequently in coastal waters across the world. Recently, new taxonomic information has enriched the known species diversity of the complex from 3 to 7 taxa. However, the identities of many species comprising the complex in China is still unclear. To clarify the species diversity and provide solid identification criteria for further studies, 332 monoclonal strains belonging to the C. lorenzianus complex were collected from the coast of China. With light microscopy and scanning and transmission electron microscopy, the morphologies of vegetative cells and resting spores were observed. Hypervariable D1-D3 regions of nuclear large subunit ribosomal encoded genes were amplified to construct the phylogenetic relationship. Morphological clustering was consistent with molecular systematics, which indicated that a total of 5 species are present within the $C$. lorenzianus complex along the coast of China; C. decipiens, C. elegans, C. laevisporus, C. mannaii and C. pauciramosus. The ultrastructure on setae, such as the shape, size and density of the setae pores, can be used as distinguishing features among allied taxa. The morphology of the resting spores also aid in accurate identification. The previous reports identifying the taxa within the $C$. lorenzianus complex in China need to be reexamined. Chaetoceros decipiens and $C$. laevisporus are the most widespread taxa along the coast of China, whereas C. pauciramosus has the narrowest distribution, having only been recorded in Taishan and Zhuhai of the Guangdong Province.
\end{abstract}

收稿日期: 2018-09-28; 接受日期: 2019-01-27

基金项目: 国家自然科学基金(31570205)和科技基础资源调查专项(2018FY100200)

* 通讯作者 Author for correspondence. E-mail: liyang@scnu.edu.cn 
Key words: Chaetoceros lorenzianus complex; species diversity; morphology; molecular phylogeny; resting spore; coast of China

角毛藻属(Chaetoceros) 是物种多样性最为丰富 的硅藻类群之一, 广泛分布于全球近岸水域(Round et al, 1990; Hasle \& Syvertsen, 1997)。洛氏角毛藻复 合群(C. lorenzianus complex)是指具有与洛氏角毛 藻(C. lorenzianus)相似形态学特征的物种集合, 典 型特征是: (1)直链状群体; (2)色素体多数; (3)角毛 硬且直; (4) 链端角毛异于链中角毛 (HernándezBecerril, 1996; Hasle \& Syvertsen, 1997)。以往普遍 认为该复合群仅包括 3 个物种: 洛氏角毛藻、并基角 毛藻(C. decipiens)和密特拉角毛藻 (C. mitra)。近年 基于形态学和分子生物学数据, 陆续报道了 4 个新 物种, 重塑了物种信息和种间界限(Li et al, 2017; Chen et al, 2018)。

除了密特拉角毛藻是典型的冷水种(Hasle \& Syvertsen, 1997)之外, 洛氏角毛藻和并基角毛藻在 我国近海均有广泛报道(金德祥, 1951; 朱树屏和郭 玉洁, 1957; 郭玉洁, 1963; 金德祥等, 1965; 郭玉洁 和钱树本, 2003; 王艳等, 2010), 是浮游植物的常见 种类(林更铭和杨清良, 2007; 王艳等, 2010; 薛冰等, 2016; 杨阳等, 2016)。然, 以往研究多是基于光镜 下特征, 物种鉴定依据相对有限, 部分研究结果存 在疑问。例如洛氏角毛藻和并基角毛藻的形态特征 十分相似, 以往区分依据是: 相邻角毛基部是否具 有“并行融合”, 有则是并基角毛藻, 反之就是洛氏 角毛藻(朱树屏和郭玉洁, 1957; 金德祥等, 1965; 郭 玉洁和钱树本, 2003)。然而近年的研究发现, “相邻 角毛基部并行融合”并不是稳定特征，同一个链状 群体中存在或长或短的并行融合, 同时也存在缺失 并行融合的情况(陈作艺和李扬, 2017; Li et al, 2017), 从而导致物种信息的重叠, 以及种间界限的 模糊。那么, 我国以往关于这两个物种的报道是否 仍然有效? 它们在我国近岸海域的地理分布如何? 此外，除了洛氏角毛藻和并基角毛藻，我国还有该 复合群的哪些物种? 它们的地理分布如何? 要回 答上述问题, 需要结合当前国际最新进展, 开展二 次研究工作, 以明确该复合群真实的多样性组成。

翟梦怡等(2017)在广东沿海开展了探索性工作, 报道了洛氏角毛藻隐藏有一定的物种多样性, 但是
由于洛氏角毛藻物种信息较为混乱，因此并未获得 有效结论。在此工作基础之上，本文聚焦中国近岸 海域，基于建立的单克隆培养藻株，结合形态学特 征和分子系统学数据, 开展洛氏角毛藻复合群物种 多样性研究，以期能够明确物种信息，厘清种间界 限，更新地理分布信息，为相关研究提供有效的物 种鉴定依据。

\section{材料和方法}

\section{1 样品采集}

于2011-2018年间, 在中国沿海进行浮游植物 活体样品的持续性采样, 站位详见图1。利用浮游植 物网(孔径 $10 \mu \mathrm{m}$ )进行水平拖网, 同步测定水温、盐 度等参数。

\section{2 单克隆培养株系的建立}

以前文描述的洛氏角毛藻复合群特征为依据,

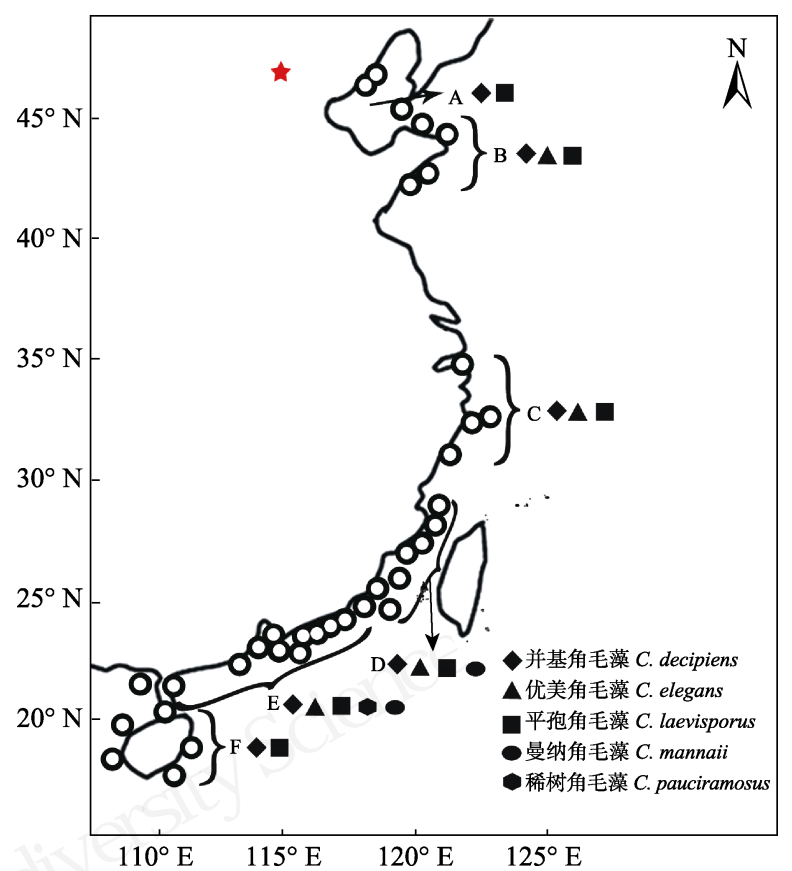

图1 采样站位及中国沿海洛氏角毛藻复合群物种地理分布 图。O: 采样站位; A: 渤海; B: 山东半岛; C: 浙江沿岸; D: 台湾海峡; E：广东沿岸; F：环海南岛。

Fig. 1 Sampling sites and geographical distribution of the Chaetoceros lorenzianus complex along the coast of China. O, Sampling sites; A, Bohai Sea; B, Shandong coast; C, Zhejiang coast; D, Taiwan Strait; E, Guangdong coast; F, Hainan coast. 
利用毛细管法在生物倒置显微镜(Mshot MI-12)下 挑取目标藻细胞, 转移至 $L$ 培养液中, 多次复洗后 建立单克隆培养株系。单克隆藻株保存于光照培养 室内, 培养条件是 $20 \pm 2^{\circ} \mathrm{C} 、 12: 12$ 光循环、光照强 度约为 $50-80 \mu \mathrm{mol}$ photonsm $\mathrm{s}^{-2} \cdot \mathrm{s}^{-1}$, 为半连续培养。 本文共建立332个单克隆培养株系。以无氮 $L$ 培养液 诱导休眠孢子(Li et al, 2017)。

\section{3 形态学特征的观察}

取对数生长期藻液, 置于光学显微镜(Olympus BX53)下进行微分干涉(differential interference contrast, DIC) 观察。电镜样品处理方法参见陈作艺和李 扬 (2017), 处理好后分别置于扫描电镜 (Zeiss Utras55)和透射电镜(Philips Tecnai10)下观察。

\section{4 基于LSU nrDNA部分序列的分子分类学分析}

用离心法收集藻细胞, 进行总DNA的提取 (Lundholm et al, 2002)。扩增核糖体大亚基编码基因 (LSU nrDNA)的D1-D3区序列。PCR产物送往深圳 华大基因公司(BGI)完成测序。利用BioEdit软件进 行序列的比对和矩阵分析 (Hall, 1999)。基于 MrModeltest 2.3 (Nylander, 2004)计算, 选择最适模 型和参数, 然后分别用 RAxML-HPC2 (http://www.phylo.org/portal2/home.action) (Miller et al, 2010)和MrBayes 3.2 (Ronquist et al, 2012)构建最 大似然树(Maximum Likelihood, ML) 和贝叶斯推理 树(Bayesian inference, BI)。其中ML分析的自检值 (bootstrap test) 设定为 1,000 。选用冕孢角毛藻 $(C$. diadema, EF423433)为群外对照。

\section{2 结果}

依据形态学特征, 如窗孔形状、壳面特征、角 毛的超微结构, 以及休眠孢子特征等, 本文建立的 株系可划分为 5 个类群, 分子系统学结论与之相一 致。综合形态学和分子系统学数据, 鉴定到我国洛 氏角毛藻复合群的 5 个物种: 并基角毛藻、优美角毛 藻(C. elegans)、平狍角毛藻(C. laevisporus)、曼纳角 毛藻(C. mannaii)和稀树角毛藻(C. pauciramosus)。 这5个物种在中国沿海的地理分布见图1。

\section{1 形态学描述}

\section{(1)并基角毛藻Chaetoceros decipiens Cleve}

常形成直链状群体; 细胞内含多个色素体; 细 胞宽环面观呈矩形(图2A)。壳面观宽椭圆形(图2C)。 链端壳面有一个中央唇形突(图2D, H)。壳面具辐射
状肋纹(图2H), 延伸至壳套, 肋纹之间散布孔纹(图 2I)。环带多数, 环带分布有平行排列的肋纹, 其间 散布一些小孔(图2J)。窗孔呈椭圆形(图2A)。相邻 角毛基部有或长或短的并行融合(图2B), 有时则无 (图2A)。角毛上分布有4-6排纵向交替排列的孔纹和 小刺, 孔纹呈椭圆形(图2F, G), 长0.3-0.6 $\mu \mathrm{m}$, 密度 为10 $\mu \mathrm{m}$ 内 $14-25$ 个, 有时光镜下可见(图2E)。壳面 长轴27.1-54.2 $\mu \mathrm{m}$, 贯壳轴8.2-18.3 $\mu \mathrm{m}$, 窗孔高 4.4-15.1 $\mu \mathrm{m}(n=50)$ 。未见休眠狍子。

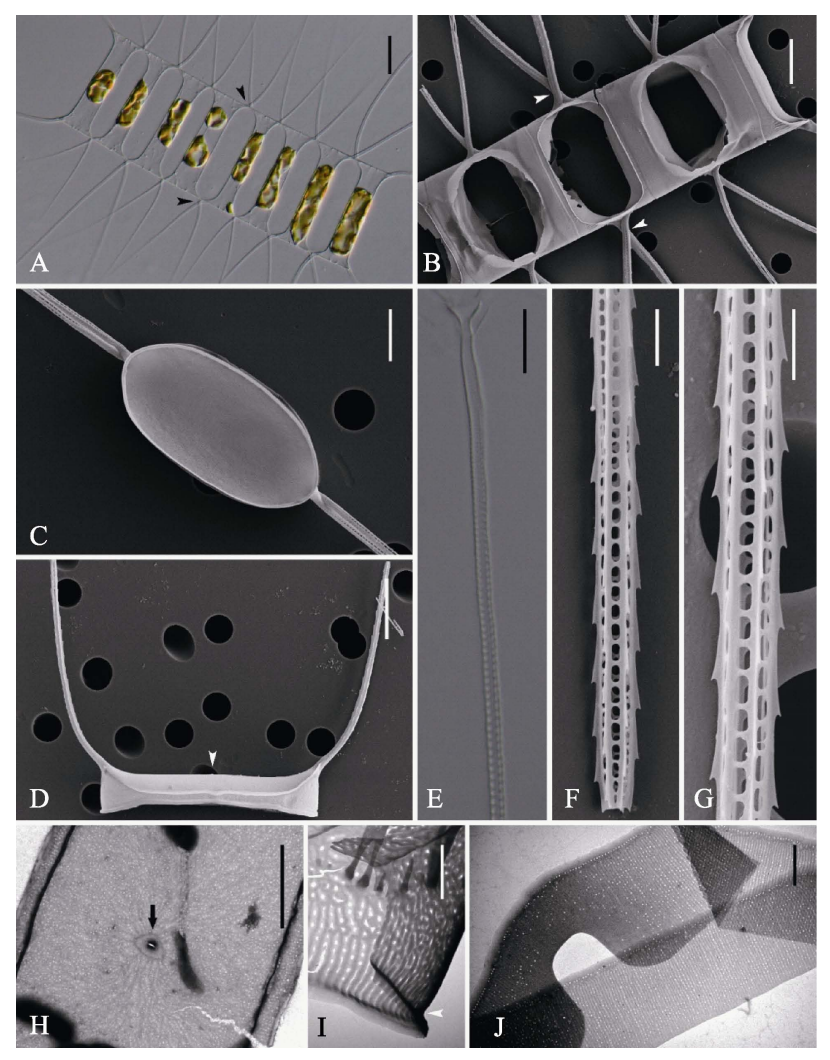

图2 并基角毛藻的光学显微镜(LM) (A, E)、扫描电镜(SEM) (B-D, F, G)和透射电镜(TEM) (H-J)示意图。A-B: 细胞链 宽环面观, 分别显示链中角毛无并行融合 (A, 箭头)及有并 行融合(B, 箭头); $C$ : 壳面观; $D$ : 链端壳面, 示 $U$ 型端角毛 及唇形突(箭头); E-G: 角毛结构; H: 链端壳面, 示唇形突 (箭型); I: 壳套; J: 环带。标尺 = $20 \mu \mathrm{m}(\mathrm{A}), 10 \mu \mathrm{m}(\mathrm{B}, \mathrm{D}$,

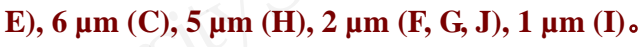

Fig. 2 Morphology of Chaetoceros decipiens under light microscopy (LM) (A, E), scanning electron microscopy (SEM) (B-D, F, G) and transmission electron microscopy (TEM) $(\mathrm{H}-\mathrm{J})$. A-B, Broad girdle views showing fusing sibling setae base present (B, arrowheads) and not (A, arrowheads); C, Internal view of intercalary valve; $\mathrm{D}$, Terminal valve with rimoportula (arrowhead) and U-shaped terminal setae; E-G, Structure of setae; $\mathrm{H}$, Terminal valve with rimoportula (arrow); I, Mantle; J, Girdle bands. Scale bars, $20 \mu \mathrm{m}(\mathrm{A}), 10 \mu \mathrm{m}$ (B, D, E), $6 \mu \mathrm{m}$ (C), $5 \mu \mathrm{m}$ (H), $2 \mu \mathrm{m}$ (F, G, J), $1 \mu \mathrm{m}$ (I). 
分布：本文标本采自海南东方市(11月)、儋州市 (11月)、文昌市(11月)、三亚湾(11月)，广西涠洲岛 (5月), 广东柘林湾(4月)、南澳岛(3月)、台山(8月)、 大亚湾(1、3、12月)、万山群岛(4、5、10、12月)、 珠海(8月)、湛江(8月), 香港(4、7、12月), 福建厦 门(5月), 台湾海峡(3、4、5、7月), 浙江宁波(8、11 月)、温州(7月)，山东烟台(5月)、威海(5月)、青岛 胶州湾(5、9月)，以及河北秦皇岛(6、7、8月)(图1)。

(2)优美角毛藻Chaetoceros elegans Yang Li, Boonprakob, Moestrup \& Lundholm

细胞链直; 细胞内含 4-10个色素体; 细胞宽环 面观呈矩形(图3A)。壳面观宽椭圆形(图3C)。壳面 分布辐射状硅质肋纹(图3G), 延伸至壳套, 肋纹之 间散布孔纹(图3F)。环带多数, 环带分布有平行排 列的肋纹, 其间散布一些小孔(图3H)。窗孔大而圆, 呈四边形(图3A, C)。角毛基部明显, 先沿着贯壳轴 方向延伸，后逐渐与之垂直(图3C)。角毛上分布有 4-6排纵向交替排列的孔纹和小刺, 孔纹呈水滴形 (图3J)或椭圆形(图3I)，长0.3-1.6 $\mu \mathrm{m}$, 密度为10 $\mu \mathrm{m}$ 内6-27个。链端壳面有一个中央唇形突, 外管短(图 3E)。壳面长轴13.2-35.8 $\mu \mathrm{m}$, 贯壳轴9.4-38.3 $\mu \mathrm{m}$, 窗孔高5.2-13.1 $\mu \mathrm{m}(n=50)$ 。休眠孢子位于母细胞 中央(图3B)。初生壳面上有两个雉状突起, 顶生多 个二叉分支, 5-6级分叉, 较浓密, 分支末端呈倒钩 状。次生壳面有一或两个驼峰状结构(图3D)。

分布: 本文标本采自广东大亚湾(3、11月)、万 山群岛(12月)、台山 (8月)、珠每 (8月)、湛工 (8月), 香 港(3、4、7、9、12月), 福建厦门(7月), 台湾海峡(3、 7月), 浙江宁波(8、11月)及山东青岛胶州湾(5、9 月)(图1)。

(3)平孢角毛藻Chaetoceros laevisporus Yang Li, Boonprakob, Moestrup \& Lundholm

细胞链直, 壳面硅质化略重; 细胞内含多个色 素体; 细胞宽环面观呈矩形(图4A)。壳面观宽椭圆 形(图4C)。壳面分布辐射状硅质肋纹(图4G), 延伸 至壳套(图4F)。环带多数, 环带分布有平行排列的 肋纹, 未见小孔(图4H)。窗孔随圆形(图4A)。角毛 上分布有4-6排纵向交替排列的孔纹和小刺, 孔纹 呈粗圆形(图4I, J), 长0.3-0.9 $\mu \mathrm{m}$, 密度为 $10 \mu \mathrm{m}$ 内 11-17个。链端壳面有一个中央唇形突, 无外管, 有 时周围有许多小刺(图4E)。壳面长轴26.5-35.3 $\mu \mathrm{m}$, 贯壳轴13.2-32.1 $\mu \mathrm{m}$, 窗孔高6.3-12.6 $\mu \mathrm{m}(n=50)$ 。

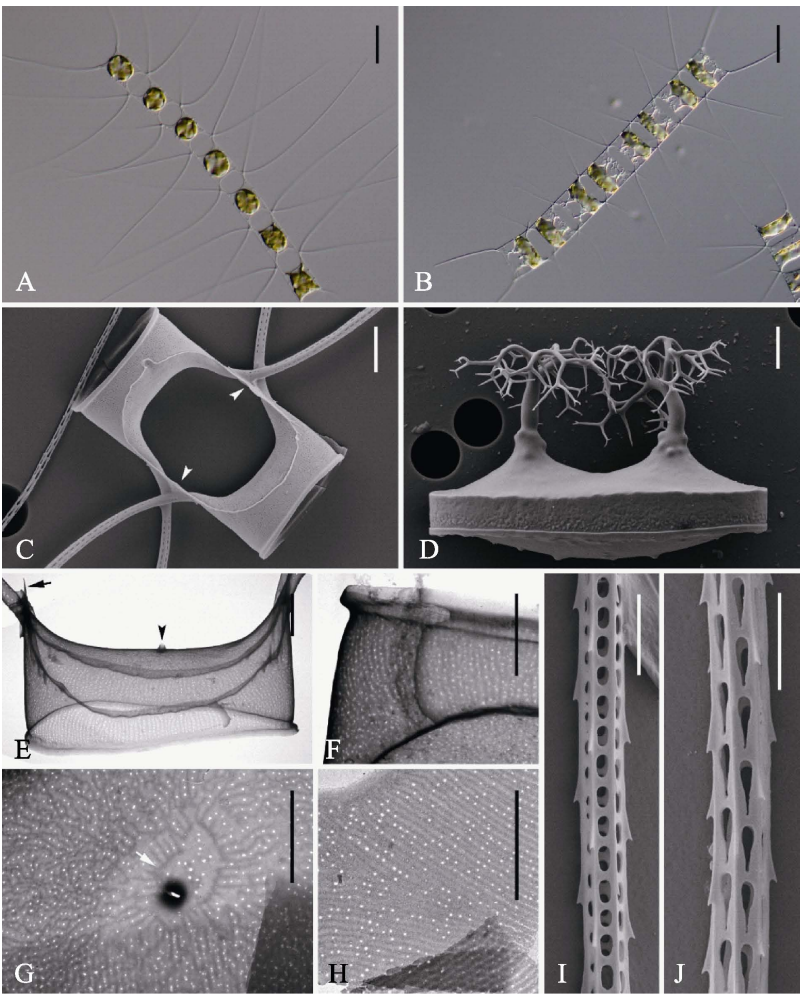

图3 优美角毛藻的光学显微镜(LM) (A-B)、扫描电镜(SEM) (C-D, I-J)和透射电镜(TEM) (E-H)示意图。A: 链状群体; B: 休眠孢子位于母细胞链中; C: 链中壳面, 示硅质翼(箭 头); $\mathrm{D}$ : 释放的成熟休眠孢子; $\mathrm{E}$ : 端壳面, 示唇形突(箭头) 和硅质脊(箭型); F: 壳套; G: 链端壳面, 示中央环纹(箭型); H: 环带; I-J: 角毛结构. 标尺 = $20 \mu \mathrm{m}(\mathrm{A}, \mathrm{B}), 6 \mu \mathrm{m}$ (D), 4 $\mu \mathrm{m}$ (C), $2 \mu \mathrm{m}$ (E, F, G, H, I, J)。

Fig. 3 Morphology of Chaetoceros elegans under light microscopy (LM) (A-B), scanning electron microscopy (SEM) (C-D, I-J) and transmission electron microscopy (TEM) (E-H). A, Chain in broad girdle view; B, Resting spores within the mother cells of a chain; C, Sibling intercalary valves showing overlapping silica ear-like structures (arrowhead); D, Released resting spore. E, Terminal valve with external process of rimoportula (arrowhead) and fringe (arrow); F, Parallel rows of poroids on the mantle; G, Central annulus (arrow) on terminal valve; H, Girdle bands; I and J, Seta structure. Scale bars, 20 $\mu \mathrm{m}$ (A, B), $6 \mu \mathrm{m}$ (D), $4 \mu \mathrm{m}$ (C), $2 \mu \mathrm{m}$ (E, F, G, H, I, J).

休眠孢子位于母细胞中央(图4B), 壳面光滑(图4B, D)。初生壳上有两个驼峰状突起, 次生壳也有 $1-2$ 个突起(图4D)。

分布：本文标本采自海南省东方市(11月)，广 东柘林湾(4月)、南澳岛(5、10月)、台山(8月)、珠 海(11月)、大亚湾(1、5、10、11、12月)、万山群岛 (5、11、12月), 香港( $4 、 7 、 12$ 月), 福建厦门(5月), 台 湾海峡(3、7月), 浙江宁波(8月)、温州(7月), 山东 青岛胶州湾(5、9月)、烟台(5月)，河北秦皇岛(6、7、 8月), 以及辽宁大连的獐子岛(9月)(图1)。 


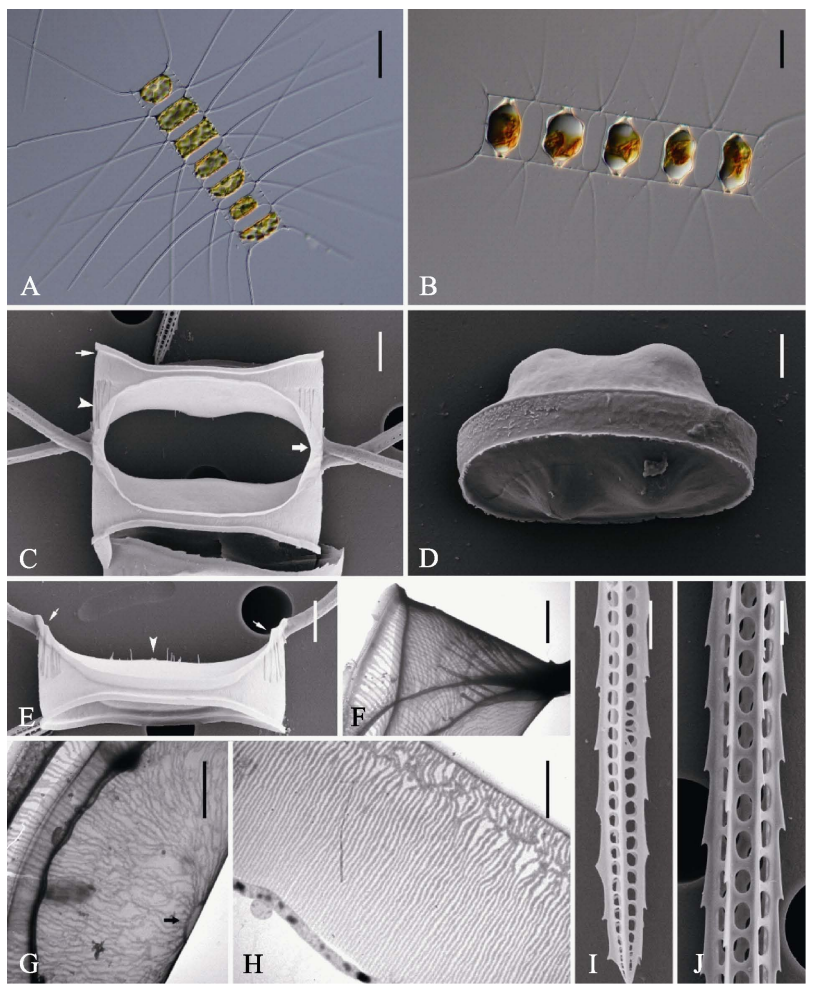

图4 平孢角毛藻的光学显微镜(LM) (A-B)、扫描电镜(SEM) (C-E, I-J)和透射电镜(TEM) (F-H)示意图。A: 链状群体; B: 休眠孢子位于母细胞链中; C: 链中壳面, 示硅质翼(宽箭型), 硅质脊(箭头)及壳套基部凹槽(窄箭型); $\mathrm{D}$ : 释放的成熟休眠 孢子; $\mathrm{E}$ : 链端壳面, 示唇形突(箭头)及硅质肋纹(箭型); F: 壳套; G: 链中壳面, 示中央环纹(箭型); H: 环带; I, J: 角 毛结构。标尺 = $50 \mu \mathrm{m}$ (A), $20 \mu \mathrm{m}$ (B), $4 \mu \mathrm{m}$ (C, E), $2 \mu \mathrm{m}$ (D, F, G, H, I, J)。

Fig. 4 Morphology of Chaetoceros laevisporus under light microscopy (LM) (A-B), scanning electron microscopy (SEM) (C-E, I-J) and transmission electron microscopy (TEM) (F-H). A, Chain in broad girdle view; B, Resting spores within the mother cells of a chain; C, Sibling intercalary valves showing overlapping silica wings (broad arrow), silica ridges (arrowhead) and furrow above the basal ring of mantle (arrow); $\mathrm{D}$, A released resting spore; E, Terminal valve with central processes (arrowhead) and silica rib (arrows); F, Mantle; G, Intercalary valve with central annulus (arrow); $\mathrm{H}$, Girdle bands; I and J, Setae structure. Scale bars, $50 \mu \mathrm{m}$ (A), $20 \mu \mathrm{m}$ (B), 4 $\mu \mathrm{m}(\mathrm{C}, \mathrm{E}), 2 \mu \mathrm{m}$ (D, F, G, H, I, J).

\section{(4)曼纳角毛藻Chaetoceros mannaii Boonpra-} kob, Yang Li, Moestrup \& Lundholm

细胞链直; 细胞内含多个色素体; 细胞宽环面 观呈矩形(图5A)。壳面观宽椭圆形(图5E)。壳面分 布辐射状硅质肋纹(图5F), 延伸至壳套(图5G)。环带 多数, 环带分布有平行排列的肋纹, 其间散布一些 小孔(图5H)。窗孔呈六边形(图5A, B)。角毛基部短 (图5D)。角毛上分布有4-6排纵向交替排列的孔纹和 小刺, 孔纹呈椭圆形(图5J), 长0.8-1.5 $\mu \mathrm{m}$, 密度为

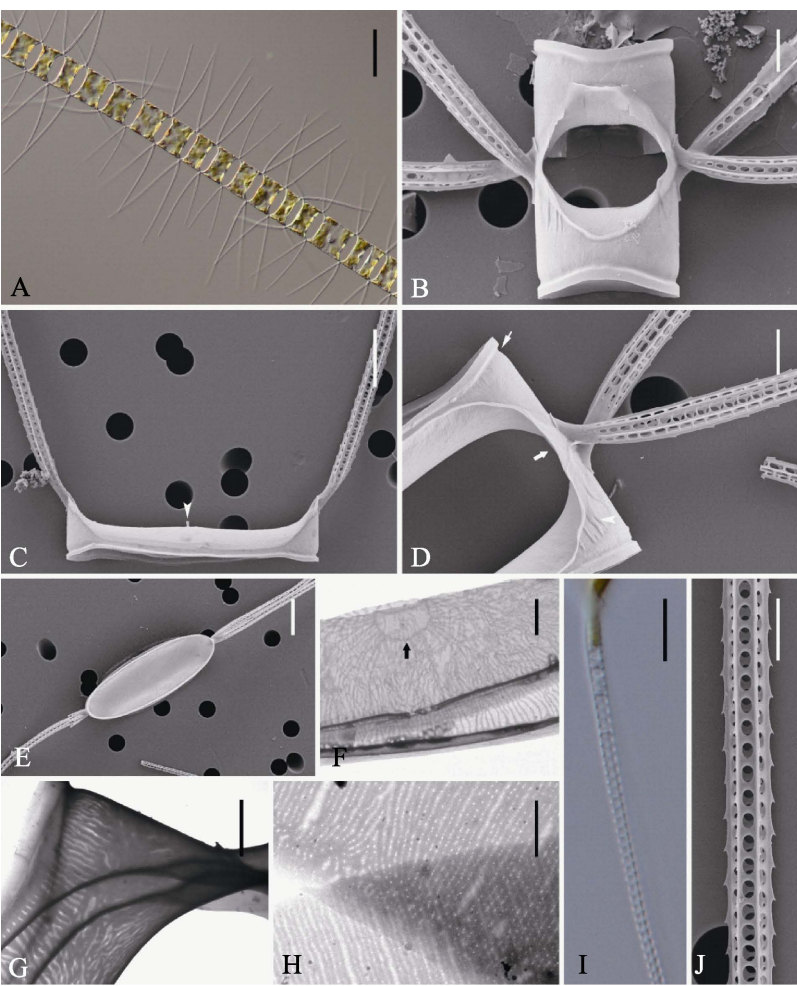

图5 曼纳角毛藻的光学显微镜(LM) (A, I)、扫描电镜(SEM) (B-E, J)和透射电镜(TEM) (F-H)示意图。A: 链状群体; B: 链中壳面; C: 端壳面, 示唇形突(箭头); D: 链中壳面, 示重 叠硅质翼(宽箭型), 硅质脊(箭头)及壳套基部凹槽(窄箭型); E-F: 链中壳面, 示中央环纹(F, 箭型); G: 壳套; H: 环带; I, $\mathrm{J}$ : 角毛结构。标尺 = $50 \mu \mathrm{m}(\mathrm{A}), 10 \mu \mathrm{m}(\mathrm{B}, \mathrm{D}, \mathrm{I}), 5 \mu \mathrm{m}(\mathrm{F}), 4$ $\mu \mathrm{m}(\mathrm{C}, \mathrm{E}, \mathrm{J}), 2 \mu \mathrm{m}(\mathrm{G}, \mathrm{H})$ 。

Fig. 5 Morphology of Chaetoceros mannaii under light microscopy (LM) (A, I), scanning electron microscopy (SEM) (B-E, J) and transmission electron microscopy (TEM) (F-H). A, Chain in broad girdle view; $\mathrm{B}$, Sibling intercalary valves showing aperture; C, Terminal valve view with external process of rimoportula (arrowhead); D, Intercalary cells with overlapping silica wings (broad arrow), silica ridges (arrowhead) and furrow above the basal ring of mantle (arrow); E, F, Internal view of an intercalary valves, showing central annulus (F, arrow); G, Mantle; H, Girdle bands; I and J: Setae structure. Scale bars, $50 \mu \mathrm{m}$ (A), $10 \mu \mathrm{m}$ (B, D, I), $5 \mu \mathrm{m}$ (F), 4 $\mu \mathrm{m}(\mathrm{C}, \mathrm{E}, \mathrm{J}), 2 \mu \mathrm{m}(\mathrm{G}, \mathrm{H})$.

$10 \mu \mathrm{m}$ 内 $6-10$ 个, 光镜下可见(图5I)。链端壳面有一 个中央唇形突, 外管长 (图5C)。壳面长轴20.0$47.8 \mu \mathrm{m}$, 贯壳轴10.4-21.0 $\mu \mathrm{m}$, 窗孔高8.3-15.2 $\mu \mathrm{m}$ $(n=50)$ 。末见休眠孢子。

分布: 本文标本采自台湾海峡(3、4月)和南海 北部海域(5、6月)(图1)。

(5)稀树角毛藻Chaetoceros pauciramosus Z. Y. Chen, N. Lundholm, Ø. Moestrup, J. Kownacka and Yang Li

细胞链直; 细胞内含4-6个色素体; 细胞宽环 
面观呈四边形(图6A)。壳面观呈宽椭圆形(图6C)。 壳面分布辐射状硅质肋纹(图6G), 延伸至壳套, 肋 纹之间散布孔纹(图6F)。环带多数, 环带分布有平 行排列的肋纹，其间散布一些小孔(图6H)。窗孔呈 六边形或花生形; 角毛基部短而不明显; 角毛基部 有并行融合现象, 或长或短, 或无(图6A，C)。角毛 上分布有4-6排纵向交替排列的孔纹和小刺，孔纹 呈细长形(图6I, J), 长0.1-0.6 $\mu \mathrm{m}$, 密度为 $10 \mu \mathrm{m}$ 内 18-44个。链端壳面有一个中央唇形突，外管短
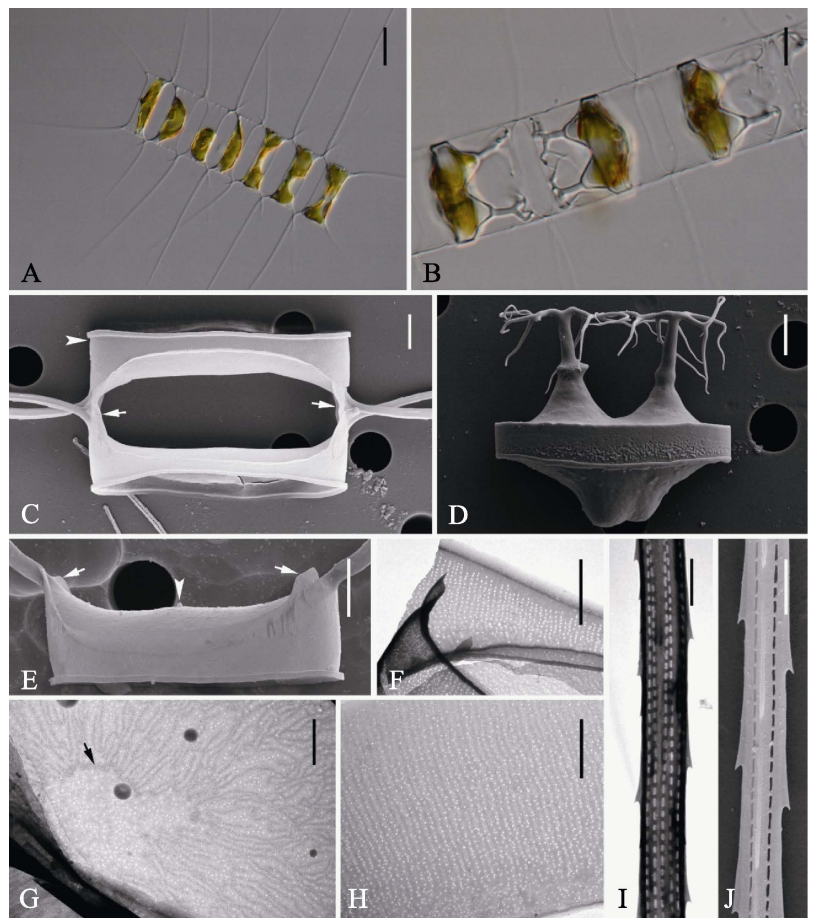

图6 稀树角毛藻的光学显微镜(LM) (A-B)、扫描电镜(SEM) (C-D, I-J)和透射电镜(TEM) (E-H)示意图。A: 链状群体; B: 休眠孢子位于母细胞链中; C: 链中壳面, 示重叠硅质翼 (箭型)及壳套基部凹槽(箭头); $\mathrm{D}$ : 释放的成熟休眠孢子; $\mathrm{E}$ : 链端壳面, 示中央唇形突(箭头)和硅质肋纹(箭型); F: 壳套; G: 链中壳面, 示中央环纹(箭型); H: 环带; I-J: 角毛结构。 标尺 = $20 \mu \mathrm{m}$ (A), $10 \mu \mathrm{m}$ (D), $4 \mu \mathrm{m}$ (C, D, E), $2 \mu \mathrm{m}$ (F), 1 $\mu \mathrm{m}$ (G, H, I, J)。

Fig. 6 Morphology of Chaetoceros pauciramosus under light microscopy (LM) (A-B), scanning electron microscopy (SEM) (C-D, I-J) and transmission electron microscopy (TEM) (E-H). A, Chain in broad girdle view; B, Resting spores within the mother cells of a chain; C, Sibling intercalary valves showing overlapping silica ear-like structures (arrows) and furrow above the basal ring of mantle (arrowhead); D, Released resting spore; E, Terminal valve with short external tubes of rimoportulae (arrowhead) and silica rib (arrows); F, Mantle; G, Intercalary valve, showing central annulus (arrow); H, Girdle bands; I and $\mathrm{J}$, Seta structure. Scale bars, $20 \mu \mathrm{m}$ (A), $10 \mu \mathrm{m}$ (D), $4 \mu \mathrm{m}$ (C, D, E), $2 \mu \mathrm{m}$ (F), $1 \mu \mathrm{m}$ (G, H, I, J).
(图6E)。壳面长轴12.5-35.4 $\mu \mathrm{m}$, 贯壳轴高8.3$24.0 \mu \mathrm{m}$, 窗孔高4.2-10.4 $\mu \mathrm{m}(n=50)$ 。休眠孢子位 于母细胞中央(图6B)。初生壳面上有两个雉状突起, 顶生多个二叉分支，2-3级分叉，分支末端呈尖刺状 结构。次生壳面有1-2个驼峰状结构(图6D)。

分布: 本文标本采自广东台山(8月)和珠海(8月) (图1)。

\section{2 基于LSU nrDNA D1-D3区的分子系统学分析}

选取了59个代表性LSU nrDNA D1-D3序列，用 于洛氏角毛藻复合群系统学关系的构建, 其中45个 来自本研究，另外14个下载自美国国立生物技术信 息中心(National Center for Biotechnology Information, NCBI)。以冕狍角毛藻(EF423433)为外类群, 分 别构建ML和BI系统进化树。因两者具有相似的拓扑 结构, 继而将两者合并(图7)。分子系统树显示(图7), 59 个目标藻株聚成 6 个类群, 均具有较高的ML和BI 置信值。分子聚类支持形态学鉴定结果。平狍角毛 藻位于洛氏复合群的最外分支(Clade I, BI $=1.00$, $\mathrm{ML}=96, \mathrm{KX} 065240$ 为模式株系), 其次分离出来的 是密特拉角毛藻(Clade II, BI $=1.00, M L=74)$ 。再向 内是曼纳角毛藻 (Clade III, BI $=1.00, \mathrm{ML}=99$, KX065246为模式株系), 以及并基角毛藻(Clade IV, $\mathrm{BI}=1.00, \mathrm{ML}=85, \mathrm{KX} 065223$ 为异模株系)。优美角 毛藻(Clade V, BI = 1.00, ML = 74, KX065232为模式 株系)和稀树角毛藻(Clade VI, BI $=1.00, M L=99$, MG867583为模式株系)位于最内侧。

基于LSU nrDNA D1-D3序列碱基，分析了各 个物种之间的遗传距离和碱基差异数(表1)。并基角 毛藻与优美角毛藻的亲缘关系最近, 遗传距离为 0.027 , 碱基差异数为 17 个, 两者的共享形态特征表 现为壳面及壳套均具有孔纹。稀树角毛藻与并基角 毛藻、优美角毛藻的亲缘关系也比较近, 遗传距离 均为 0.028 , 碱基差异数分别为 18 个和 21 个, 稀树角 毛藻和并基角毛藻的共享特征是具有相邻角毛基 部的并行融合, 而稀树角毛藻和优美角毛藻的共享 特征是具有二叉分支的休眠孢子。平孢角毛藻与其 他物种的遗传距离均最远，其中与曼纳角毛藻的遗 传距离最远, 为 0.105 , 碱基差异数为 69 个。平孢角 毛藻的区别特征是休眠孢子仅具有隆起，但不具有 突起及分支结构。 


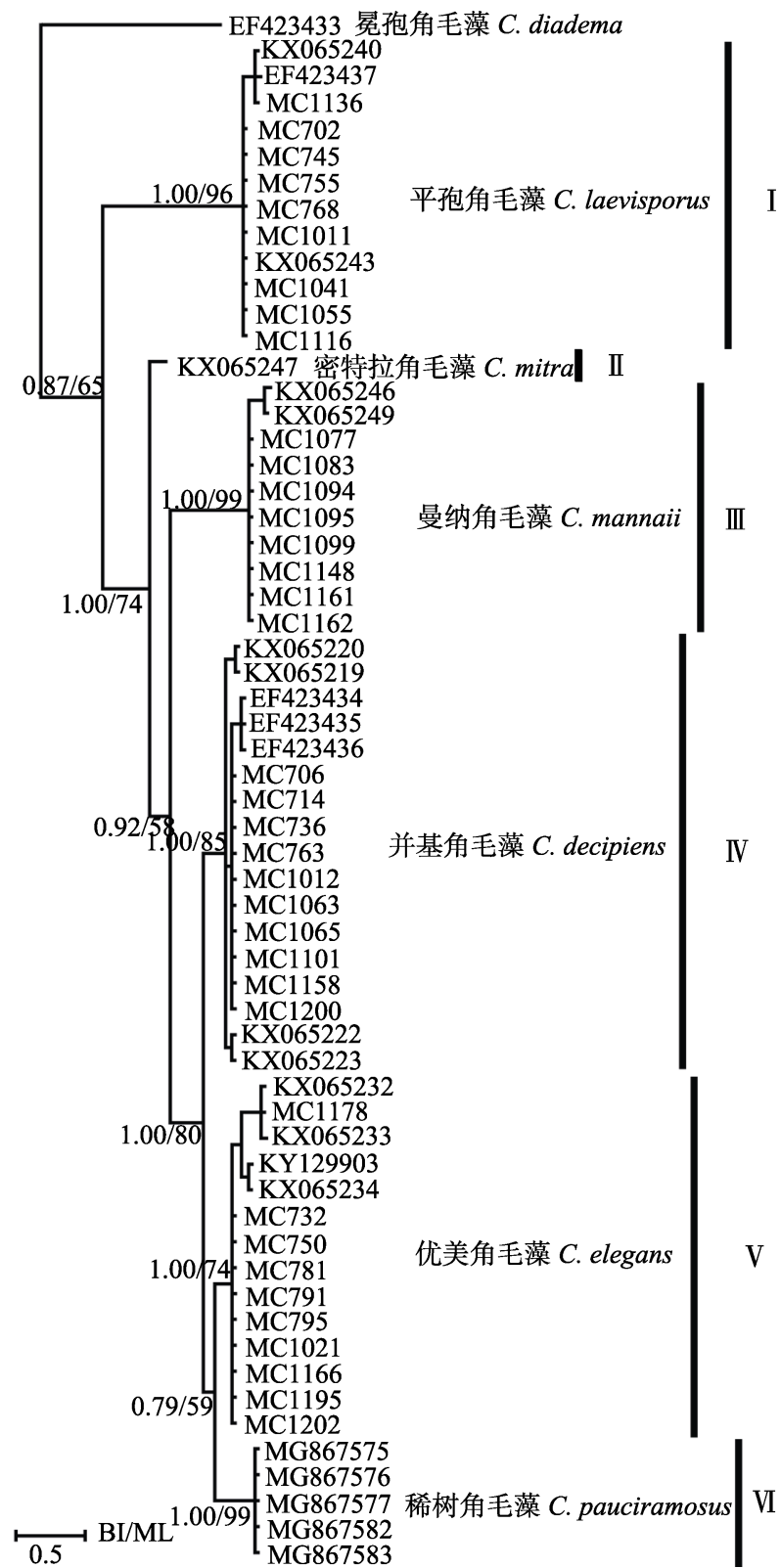

图7 基于核糖体大亚基部分序列的最大似然树。分支上的 置信值分别显示贝叶斯分析和最大似然分析。

Fig. 7 Molecular phylogenetic tree inferred from LSU rDNA, with Chaetoceros diadema as outgroup. Supporting values on each nodule are from Bayesian and Maximum Likelihood analysis.

\section{3 讨论}

\section{1 相邻物种的形态学比较}

以往关于角毛藻的分类学工作多局限于光镜 特征, 如群体形态、角毛走势、窗孔形态等, 被认 为是重要的分类学特征, 而电镜技术的应用相对较 少。此外, 以往的形态学观察集中于自然样品, 较 少开展基于培养株系的形态学工作，因此存在将自 然样品中不同物种的特征错误归入同一物种的情 况，继而导致物种信息的混乱(Evesen \& Hasle, 1975; Rines \& Hargraves, 1988; Jensen \& Moestrup, 1998; Kooistra et al, 2010)。随着电镜技术的广泛应 用，以及分子生物学信息的补充，一些以往常用的 光镜特征，如群体形态、角毛走势等，已被证实是 易变特征, 不能作为有效分类依据(陈作艺和李扬, 2017)。目前被广泛认可的有效分类特征有：角毛上 的超微结构、壳面及壳套有无孔纹、休眠孢子特征 等, 尤其是角毛上的超微结构以及休眠孢子特征被 赋予重要的分类学价值(Li et al, 2017)。

近年来，随着新物种的陆续报道，洛氏角毛藻 复合群的物种组成已增加至7个(Li et al, 2017; Chen et al, 2018), 各个物种的识别信息都得到了确认和 完善。密特拉角毛藻是典型的冷水种，主要分布在 北大西洋海域(Hasle \& Syvertsen, 1997; Li et al, 2017)。该种角毛相对柔软，向各个方向延伸，链中 角毛呈Brunel II型，该特征区别于复合群内其他物 种(Brunel I型)(Li et al, 2017)。此外, 密特拉角毛藻 的角毛孔纹最为细密(表2)。洛氏角毛藻复合群中, 并基角毛藻的报道最为广泛，全球各个海域均有分 布，该种因链中角毛基部存在并行融合而得名。然 而近年研究发现角毛基部的并行融合特征并不稳 定，易受环境条件、生活史阶段的影响(陈作艺和李 扬, 2017; Li et al, 2017)。目前该种认可的区别特征

表1 洛氏角毛藻复合群中各个物种之间的遗传距离(括号内为碱基差异数)

Table 1 Genetic distance among allied taxa within the Chaetoceros lorenzianus complex (data in the brackets are numbers of different base pairs)

\begin{tabular}{llllll}
\hline & $\begin{array}{l}\text { 平狍角毛藻 } \\
\text { C. laevisporus }\end{array}$ & $\begin{array}{l}\text { 密特拉角毛藻 } \\
\text { C. mitra }\end{array}$ & $\begin{array}{l}\text { 曼纳角毛藻 } \\
\text { C. mannaii }\end{array}$ & $\begin{array}{l}\text { 并基角毛藻 } \\
\text { C. decipiens }\end{array}$ & $\begin{array}{l}\text { 优美角毛藻 } \\
\text { C. elegans }\end{array}$ \\
\hline 密特拉角毛藻 C. mitra & $0.081(52)$ & & & & \\
曼纳角毛藻 C. mannaii & $0.105(69)$ & $0.047(33)$ & & & \\
并基角毛藻 C. decipiens & $0.089(58)$ & $0.028(20)$ & $0.057(38)$ & & $0.027(17)$ \\
优美角毛藻 C. elegans & $0.083(53)$ & $0.036(25)$ & $0.067(44)$ & $0.028(18)$ & $0.028(21)$ \\
稀树角毛藻 C. pauciramosus & $0.092(60)$ & $0.040(26)$ & $0.059(39)$ & & \\
\hline
\end{tabular}


表2 洛氏角毛藻复合群相似物种之间的形态学比较

Table 2 Morphological comparison among allied taxa within Chaetoceros lorenzianus complex

\begin{tabular}{|c|c|c|c|c|c|c|c|}
\hline $\begin{array}{l}\text { 特征 } \\
\text { Character }\end{array}$ & $\begin{array}{l}\text { 并基角毛藻 } \\
\text { C. decipiens }\end{array}$ & $\begin{array}{l}\text { 优美角毛藻 } \\
\text { C. elegans }\end{array}$ & $\begin{array}{l}\text { 平孢角毛藻 } \\
\text { C. laevisporus }\end{array}$ & $\begin{array}{l}\text { 曼纳角毛藻 } \\
\text { C. mannaii }\end{array}$ & $\begin{array}{l}\text { 稀树角毛藻 } \\
\text { C. pauciramosus }\end{array}$ & $\begin{array}{l}\text { 密特拉角毛藻 } \\
\text { C. mitra }\end{array}$ & $\begin{array}{l}\text { 洛氏角毛藻 } \\
C . \text { lorenzianus } \\
\text { type material }\end{array}$ \\
\hline $\begin{array}{l}\text { 角毛孔纹形状 } \\
\text { Seta poroid shape }\end{array}$ & $\begin{array}{l}\text { 椭圆形 } \\
\text { Oval }\end{array}$ & $\begin{array}{l}\text { 水滴状 } \\
\text { Drop-shaped }\end{array}$ & $\begin{array}{l}\text { 椭圆形 } \\
\text { Oval }\end{array}$ & $\begin{array}{l}\text { 椭圆形 } \\
\text { Oval }\end{array}$ & $\begin{array}{l}\text { 细长形 } \\
\text { Elongated }\end{array}$ & $\begin{array}{l}\text { 圆形或籿圆形 } \\
\text { Round or oval }\end{array}$ & $\begin{array}{l}\text { 圆形 } \\
\text { Oval }\end{array}$ \\
\hline $\begin{array}{l}\text { 角毛孔纹大小 } \\
\text { Seta poroid size }(\mu \mathrm{m})\end{array}$ & $\begin{array}{l}0.3-0.6 \\
(0.4 \pm 0.1)\end{array}$ & $\begin{array}{l}0.3-1.6 \\
(0.7 \pm 0.3)\end{array}$ & $\begin{array}{l}0.3-0.9 \\
(0.6 \pm 0.1)\end{array}$ & $\begin{array}{l}0.8-1.5 \\
(1.1 \pm 0.1)\end{array}$ & $\begin{array}{l}0.1-0.6 \\
(0.3 \pm 0.1)\end{array}$ & $\begin{array}{l}0.1-0.3 \\
(0.2 \pm 0.1)\end{array}$ & $\begin{array}{l}\mathrm{Nd} \\
\mathrm{Nd}\end{array}$ \\
\hline $\begin{array}{l}\text { 角毛孔纹密度 } \\
\text { Seta poroid number } \\
\text { in } 10 \mu \mathrm{m}\end{array}$ & $\begin{array}{l}14-25 \\
(20.2 \pm 3.6)\end{array}$ & $\begin{array}{l}6-27 \\
(18.7 \pm 5.0)\end{array}$ & $\begin{array}{l}11-17 \\
(14.1 \pm 1.9)\end{array}$ & $\begin{array}{l}6-10 \\
(7.9 \pm 1.0)\end{array}$ & $\begin{array}{l}18-44 \\
(31.7 \pm 5.4)\end{array}$ & $\begin{array}{l}30-56 \\
(39.8 \pm 7.4)\end{array}$ & $\begin{array}{l}5-9 \\
(7.2 \pm 1.7)\end{array}$ \\
\hline $\begin{array}{l}\text { Brunel 型 } \\
\text { Brunel group }\end{array}$ & $\begin{array}{l}\text { I } \\
\text { I }\end{array}$ & $\begin{array}{l}\text { I } \\
\text { I }\end{array}$ & $\begin{array}{l}\text { I } \\
\text { I }\end{array}$ & $\begin{array}{l}\text { I } \\
\text { I }\end{array}$ & $\begin{array}{l}\text { I } \\
\text { I }\end{array}$ & $\begin{array}{l}\text { II } \\
\text { II }\end{array}$ & $\begin{array}{l}\text { I } \\
\text { I }\end{array}$ \\
\hline $\begin{array}{l}\text { 角毛基部并行融合 } \\
\text { Fusion of seta bases }\end{array}$ & $\begin{array}{l}\text { 有或无 } \\
\text { Present/absent }\end{array}$ & $\begin{array}{l}\text { 无 } \\
\text { Absent }\end{array}$ & $\begin{array}{l}\text { 无 } \\
\text { Absent }\end{array}$ & $\begin{array}{l}\text { 无 } \\
\text { Absent }\end{array}$ & $\begin{array}{l}\text { 短或无 } \\
\text { Short/absent }\end{array}$ & $\begin{array}{l}\text { 无 } \\
\text { Absent }\end{array}$ & $\begin{array}{l}\text { 有 } \\
\text { Present }\end{array}$ \\
\hline $\begin{array}{l}\text { 休眠孢子 } \\
\text { Resting spore }\end{array}$ & $\begin{array}{l}\text { 未发现 } \\
\text { Unknown }\end{array}$ & $\begin{array}{l}\text { 有二叉分支 } \\
\text { Two branching } \\
\text { processes }\end{array}$ & $\begin{array}{l}\text { 壳面平滑 } \\
\text { Smooth }\end{array}$ & $\begin{array}{l}\text { 未发现 } \\
\text { Unknown }\end{array}$ & $\begin{array}{l}\text { 有二叉分支 } \\
\text { Two branching } \\
\text { processes }\end{array}$ & $\begin{array}{l}\text { 有二叉分支 } \\
\text { Two branching } \\
\text { processes }\end{array}$ & $\begin{array}{l}\text { 有二叉分支? } \\
\text { Two branching } \\
\text { processes? }\end{array}$ \\
\hline $\begin{array}{l}\text { 窗孔形状 } \\
\text { Aperture shape }\end{array}$ & $\begin{array}{l}\text { 椭圆形 } \\
\text { Oval }\end{array}$ & $\begin{array}{l}\text { 圆形或四边形 } \\
\text { Rounded or } \\
\text { quadrangular }\end{array}$ & $\begin{array}{l}\text { 粗圆形 } \\
\text { Oval }\end{array}$ & $\begin{array}{l}\text { 六边形 } \\
\text { Hexagonal }\end{array}$ & $\begin{array}{l}\text { 六边形或花生形 } \\
\text { Hexagonal or } \\
\text { peanut shaped }\end{array}$ & $\begin{array}{l}\text { 六边形或花生形 } \\
\text { Hexagonal or } \\
\text { peanut shaped }\end{array}$ & $\begin{array}{l}\text { 圆形或六边形 } \\
\text { Oval or hexagonal }\end{array}$ \\
\hline $\begin{array}{l}\text { 角毛基部 } \\
\text { Basal part of setae }\end{array}$ & $\begin{array}{l}\text { 无 } \\
\text { Lacking }\end{array}$ & $\begin{array}{l}\text { 有且明显 } \\
\text { Distinct }\end{array}$ & $\begin{array}{l}\text { 无 } \\
\text { Lacking }\end{array}$ & $\begin{array}{l}\text { 短 } \\
\text { Short }\end{array}$ & $\begin{array}{l}\text { 短 } \\
\text { Short }\end{array}$ & $\begin{array}{l}\text { 无 } \\
\text { Lacking }\end{array}$ & $\begin{array}{l}\text { 无 } \\
\text { Lacking }\end{array}$ \\
\hline $\begin{array}{l}\text { 壳面及壳套孔纹 } \\
\text { Poroids on valve } \\
\text { face and mantle }\end{array}$ & $\begin{array}{l}\text { 有 } \\
\text { Yes }\end{array}$ & $\begin{array}{l}\text { 有 } \\
\text { Yes }\end{array}$ & $\begin{array}{l}\text { 无 } \\
\text { No }\end{array}$ & $\begin{array}{l}\text { 无 } \\
\text { No }\end{array}$ & $\begin{array}{l}\text { 有 } \\
\text { Yes }\end{array}$ & $\begin{array}{l}\text { 无 } \\
\text { No }\end{array}$ & $\begin{array}{l}\text { nd } \\
\text { nd }\end{array}$ \\
\hline
\end{tabular}

nd, 无测量数据。

nd, No data available.

主要是角毛孔纹的大小和密度(表2), 壳面和壳套分 布有孔纹也可区别于部分物种。优美角毛藻的角毛 孔纹呈水滴状, 窗孔较大, 呈四方形, 这两个特征 使之显著区别于其他物种。最为粗大的角毛孔纹出 现在曼纳角毛藻, 光镜下清晰可见; 端壳面中央唇 形突有较长的外管, 也显著区别于其他物种。此外, 曼纳角毛藻的壳面硅质化较重。平孢角毛藻的窗孔 为椭圆形至花生形, 壳面未见孔纹, 角毛孔纹较为 粗大。稀树角毛藻的区别特征是角毛孔纹细长形, 排列紧密, 仅次于密特拉角毛藻(表2)。

截至目前, 除了并基角毛藻和曼纳角毛藻之外, 其他物种均报道有休眠孢子。其中平孢角毛藻的休 眠孢子平滑, 仅具有1-2个隆起结构, 未见二叉分 支状突起, 该特征区别于其他物种。密特拉角毛藻 的休眠孢子具有二叉分支结构, 但该分支较短, 末 端呈短刺状结构。优美角毛藻的休眠孢子具有浓密 的二叉分支, 多为5-6级分叉, 分支末端呈倒钩状。 而稀树角毛藻休眠狍子的二叉分支相对稀疏, 为 2-3级分叉, 分支末端呈尖刺状(表2)。

\section{2 洛氏角毛藻的研究不足}

洛氏角毛藻也是本复合群的常见物种, 全球多 个海域均有报道(Hasle \& Syvertsen, 1997), 但近年 的研究发现该种的报道存在相互矛盾、前后不一致 的情况, 导致物种信息较为残缺, 已成为相关研究 继续推进的制约。首先, 洛氏角毛藻的模式标本信 息不清。本种首次报道于亚德里亚海(Grunow, 1863), 但原始文献中仅提供了两个营养细胞壳面 的简单手绘图, 并未指定模式标本。经过对Grunow 收集标本的逐一比对, Li等(2017)明确了洛氏角毛 藻的模式标本, 提供了较为清晰的光镜特征, 但电 镜超微特征和分子生物学信息仍然缺失。其次, 异 模标本尚未建立。由于洛氏角毛藻模式标本是封片, 不能获取电镜和分子信息, 于是建立可信的异模标 本是完善物种信息的唯一途径。近年各国学者陆续 开展了该种藻株的建立工作, 但始终未能在亚德里 亚海建立洛氏角毛藻的培养株系, 这是目前工作的 重要制约。第三，休眠孢子存在疑问。该种休眠孢 子最初报道于日本海域(Okamura，1911), 仅提供了 
具有二叉分支的休眠孢子手绘图。但是其与密特拉 角毛藻休眠孢子极为相似, 贯壳轴明显大于壳面长 轴。由于没有提供对应的营养细胞图, 所以难以确 定该休眠孢子的物种归属。此后, Hasle和Syvertsen (1997) 也提供了休眠孢子的手绘图，与 Okamura (1911)相比, 其壳面长轴/贯壳轴比例、角毛藻走势 明显不同。哪个才是真正的洛氏角毛藻休眠孢子? 目前难以判断。还有其他学者也描绘过洛氏角毛藻 休眠孢子(Evesen \& Hasle, 1975; Rines \& Hargraves, 1988; Hernández-Becerril, 1996; Hasle \& Syvertsen, 1997; Jensen \& Moestrup, 1998), 但都来自于自然样 品, 也难以确认该休眠孢子是否属于洛氏角毛藻。

基于洛氏角毛藻模式标本获取的最新光镜特 征显示(Li et al, 2017), 洛氏角毛藻具有粗大且排列 稀疏的角毛孔纹, 密度为 $10 \mu \mathrm{m}$ 内7-9个, 在该复合 群中最为稀疏。同时, 模式标本的相邻角毛还具有 一定长度的并行融合，与并基角毛藻极为相似 $(\mathrm{Li}$ et al, 2017)。然而截至目前, 洛氏角毛藻的物种信息 大部分仍然缺失, 亟需在原产地建立其藻株, 补充 和完善物种信息。

\section{3 中国沿海洛氏角毛藻复合群的组成和分布}

我国以往的分类学和生态学研究中, 根据传统 认知，即依据“角毛基部是否存在并行融合”，鉴定 到洛氏角毛藻和并基角毛藻。并且证实这两个物种 在我国海域广泛分布, 是海洋浮游植物的常见组成 (朱树屏和郭玉洁, 1958; 林更铭和杨清良, 2007; 王 艳等, 2010; 薛冰等, 2016; 杨阳等, 2016)。本文结果 也证实, 洛氏角毛藻复合群物种在我国沿海广泛分 布，但该复合群在我国海域具有较高的物种多样性， 并非局限于洛氏角毛藻和并基角毛藻。随着新的物 种信息的完善, 以及种间区别依据的建立, 需要对 以往报道物种的有效性进行二次判断。然而, 我国 关于洛氏角毛藻和并基角毛藻的记录多见于生态 学研究, 单纯的分类学研究不多, 并且仍以光镜特 征为主, 缺乏电镜下超微特征, 限制了二次分析工 作的推进。

依据本文数据, 我们构建了洛氏角毛藻复合群 物种在我国沿海的地理分布图(图1), 以期能够为后 续廓清工作提供初步资料。由图1可见, 广东沿岸具 有最高的物种多样性, 该复合群的 5 个物种均有分 布, 这或许与该海域的较高采样频次有关。其次是 台湾海峡, 分布有 4 个物种, 山东和浙江沿岸均分
布有 3 个物种，渤海和海南岛观察到 2 个物种。并基 角毛藻和平孢角毛藻在我国沿岸的分布范围最为 广泛, 6 个海域均有检出。优美角毛藻分布在中间的 4 个海域, 南北两端的渤海和海南未有检出。曼纳角 毛藻分布于两个毗邻海域: 广东沿岸和台湾海峡。 稀树角毛藻的分布最为有限, 目前仅报道于广东台 山和珠海。由于地理距离的原因, 部分海域的采样频 次和采样季节有限, 这也对物种分布造成了影响。

除了广东沿海, 稀树角毛藻在波罗的海也有广 泛分布, 并且从2003年至今, 每年秋季(9-11月)在 波罗的海南部海域引发藻华(Kownacka et al, 2013; Chen et al, 2018)。目前稀树角毛藻仅报道于上述两 个海域: 中国广东和波罗的海沿岸，该种或许是世 界广布种, 还需要后续其他海域的报道加以证实。

\section{参考文献}

Chen ZY, Li Y (2017) Preliminary study on some taxonomic puzzles of Chaetoceros decipiens Cleve. Acta Hydrobiologica Sinica, 41, 914-922. (in Chinese with English abstract) [陈作艺, 李扬 (2017) 并基角毛藻若干分类学 疑问的初步探讨. 水生生物学报, 41, 914-922.]

Chen ZY, Lundholm N, Moestrup Ø, Kownacka J, Li Y (2018) Chaetoceros pauciramosus sp. nov. (Bacillariophyceae), a widely distributed brackish water species in the $C$. lorenzianus complex. Protist, 169, 615-631.

Chin TG, Chen JH, Huang KG (1965) Marine Planktonic Diatoms from China Sea, p. 115. Shanghai Science and Technology Press, Shanghai. (in Chinese) [金德祥, 陈金环, 黄凯 歌 (1965) 中国海洋浮游硅藻类, 见115页. 上海科学技 术出版社, 上海.]

Chin TG (1951) A list of Chinese diatoms from 1847 to 1946. Amoy Fishries Bulletin, 5, 145-230. (in Chinese with English abstract) [金德祥 (1951) 中国硅藻目录. 厦门水 产学报, 5, 145-230.]

Chu SP, Kuo YC (1957) Studies on the genus Chaetoceros Ehrenberg from the fishing ground of the mackerel, Pneumatophorus japonicus (Houttuyn), off the Shantung coastal from Chefoo to Weihai. Part I. A systematic study. Oceanologia et Limnologia Sinica, 1, 27-87. (in Chinese with English abstract) [ 朱树屏, 郭玉洁 (1957) 烟台、威海鲐鱼 渔场及其附近海区角毛硅藻属的研究. I. 分类的研究. 海 洋与湖沼, 1, 27-87.]

Chu SP, Kuo YC (1958) Studies on the genus Chaetoceros Ehrenberg from the fishing ground of the mackerel, Pneumatophorus japonicus (Houttuyn), off the Shanung coast from Chefoo to Weihai. Part II. An ecological study. Oceanologia et Limnologia Sinica, 1, 167-179. (in Chinese with English abstract) [朱树屏, 郭玉洁 (1958) 烟台、威海 鲐鱼渔场及其附近海区角毛硅藻属的研究. II. 生态的研 


\section{究. 海洋与湖沼, 1, 167-179.]}

Evensen DL, Hasle GR (1975) The morphology of some Chaetoceros (Bacillariophyceae) species as seen in the electron microscopes. Nova Hedwigia, 53, 152-174.

Grunow A (1863) About some new and insufficiently known species and genera of diatoms. Negotiations of the Imperial Royal Zoological-Botanical Society in Vienna, 13, 137-162.

Guo YJ (1963) The nature of Chaetoceros flora of the Yellow Sea. Oceanologia et Limnologia Sinica, 5, 322-332. (in Chinese with English abstract) [郭玉洁 (1963) 黃海角毛 藻属(Genus Chaetoceros Ehrenberg)区系的性质. 海洋与 湖沼, 5, 322-332.]

Guo YJ, Qian SB (2003) Flora Algarum Marinarum Sinicarum, Tomus V. Bacillariophyta, No.1 Centricae, pp. 345-346. Science Press, Beijing. (in Chinese) [郭玉洁, 钱树本 (2003) 中国海藻志 (第5卷): 硅藻门 (第一册), 中心纲. 345-346页. 科学出版社, 北京.]

Hall TA (1999) BioEdit: A user-friendly biological sequence alignment editor and analysis program for Window 95/98/NT. Nucleic Acids Symposium Series, 41, 95-98.

Hasle GR, Syvertsen EE (1997) Marine diatoms. In: Identifying Marine Phytoplankton (ed. Tomas CR), pp. 5-387. Academic Press, London.

Hernández-Becerril DU (1996) A morphological study of Chaetoceros species (Bacillariophyta) from the plankton of the Pacific Ocean of Mexico. Bulletin of the Natural History Museum (Botany series), 26, 1-73.

Jensen KG, Moestrup Ø (1998) The genus Chaetoceros (Bacillariophyceae) in inner Danish coastal waters. Nordic Journal of Botany, 18, 88.

Kooistra WHCF, Sarno D, Hernández-Becerril DU, Assmy P, Prisco CD, Montresor M (2010) Comparative molecular and morphological phylogenetic analyses of taxa in the Chaetocerataceae (Bacillariophyta). Phycologia, 49, 471-500.

Kownacka J, Edler L, Gromisz S, Łotocka M, Olenina I, Ostrowska M, Piwosz K (2013) Non-indigenous species Chaetoceros cf. lorenzianus Grunow 1863- A new, predominant component of autumn phytoplankton in the southern Baltic Sea. Estuarine Coastal \& Shelf Science, 119, 101-111.

Li Y, Boonprakob A, Gaonkar CC, Kooistra WHCF, Lange CB, Hernández-Becerril DU, Chen ZY, Moestrup Ø, Lundholm N (2017) Diversity in the globally distributed diatom genus Chaetoceros (Bacillariophyceae): Three new species from warm-temperate waters. PLoS ONE, 12, 1-38.

Lin GM, Yang QL (2007) Species diversity and the distribution of micro-phytoplankton in the Taiwan Strait. Biodiversity Science, 15, 31-45. (in Chinese with English abstract) [林更 铭, 杨清良 (2007) 台湾海峡小型浮游植物的物种多样 性和分布特征. 生物多样性, 15, 31-45.]
Lundholm N, Daugbjerg N, Moestrup Ø (2002) Phylogeny of the Bacillariaceae with emphasis on the genus Pseudonitzschia (Bacillariophyceae) based on partial LSU rDNA. European Journal of Phycology, 37, 115-134.

Miller MA, Pfeiffer W, Schwartz T (2010) Creating the CIPRES science gateway for inference of large phylogenetic trees. 2010 Gateway Computing Environments Workshop (GCE), New Orleans, LA, pp. 1-8.

Nylander JAA (2004) MrModeltest v2. Program distributed by the author. Evolutionary Biology Center, Uppsala University, Uppsala, Sweden.

Okamura K (1911) Some littoral diatoms of Japan. Report Imperial Fisheries Institute Tokyo Japan. 7, 3-18.

Rines JEB, Hargraves PE (1988) The Chaetoceros Ehrenberg (Bacillariophyceae) flora of Narragansett Bay, Rhode Island, USA. Bible Phycology, 79, 5-196.

Ronquist F, Teslenko M, Mark P, Ayres DL, Darling A (2012) MrBayes 3.2: Efficient Bayesian phylogenetic inference and model choice across a large model space. Systematic Biology, 61, 539-542.

Round FE, Crawford RM, Mann DG (1990) The Diatoms: Biology and Morphology of the Genera, p. 747. Cambridge University Press, Cambridge.

Wang Y, Nie R, Li Y, Lü SH (2010) Species diversity and geographical distribution of Chaetoceros in Guangdong coast waters. Advance in Marine Science, 28, 342-352. (in Chinese with English abstract) [王艳, 聂瑞, 李扬, 吕颂辉 (2010) 广东沿海角毛藻(Chaetoceros)的种类多样性及其 地理分布. 海洋科学进展, 28, 342-352.]

Xue B, Sun J, Li TT (2016) Phytoplankton community structure of northern South China Sea in summer of 2014. Haiyang Xuebao, 8, 54-65. (in Chinese with English abstract) [薛冰, 孙军, 李婷婷 (2016) 2014年夏季南海北 部浮游植物群落结构. 海洋学报, 8, 54-65.]

Yang Y, Sun J, Guan XY, Zhai WD, Guo SJ (2016) Seasonal variation of net-phytoplankton community in Bohai Sea. Marine Science Bulletin, 35, 121131. (in Chinese with English abstract) [杨阳, 孙军, 关翔宇, 翟惟东, 郭术津 (2016) 渤海网采浮游植物群集的季节变化. 海洋通报, 35, 121-131.]

Zhai MY, Zhu SY, Chen ZY, Li Y (2017) Preliminary study on the species diversity of Chaetoceros lorenzianus complex from Guangdong coastal waters. Acta Hydrobiologica Sinica, 41, 1282-1290. (in Chinese with English abstract) [翟梦怡, 朱素英, 陈作艺, 李扬 (2017) 广东沿海洛氏角 毛藻复合群物种多样性的探究. 水生生物学报, 41, 1282-1290.] 